\title{
AN EXAMPLE ABOUT NORMALIZERS IN MAPPING CLASS GROUPS
}

\author{
JANE GILMAN $^{1}$
}

\begin{abstract}
J. Birman has raised the question as to whether every element of the mapping class group (the Teichmüller modular group) is in the normalizer of some element of finite order. In this paper elements of the mapping class group which are not in the normalizer of any element of finite order are constructed.
\end{abstract}

J. Birman has raised the question as to whether every element of the mapping class group of genus $g, M(g)$, is in the normalizer of some element of finite order (see p. 190 and p. 219, problem 26 of [1]). In this paper we find an element of $M(g), g \geqslant 3$, which is not in the normalizer of any element of finite order. The example is found by combining the methods of Raymond and Tollefson [3] with a result about the action on homology of automorphisms of a surface [2]. I want to thank J. Birman for pointing out the relevance of [3].

1. Notation and definitions. Throughout this paper $F$ will denote a compac surface of genus $g>2 ; \sim$ will denote homotopy, and $\approx$ homology. For any homeomorphism $f$ of $F, f_{\#}$ will be the induced element of $M(g)$ and $f_{*}$ the induced automorphism of the first homology group of $F . N\left(f_{\#}\right)$ and $C\left(f_{\#}\right)$ will denote, respectively, the normalizer and the centralizer of $f_{\#}$ in $M(g)$, and $N\left(f_{*}\right)$ and $C\left(f_{*}\right)$, respectively, the normalizer and the centralizer of $f_{*}$ in the group of automorphisms of the first homology group.

Let $a_{1}, \ldots, a_{g}, b_{1}, \ldots, b_{g}$ be curves whose homology classes give a canonical homology basis for $F$. For any curve $c$, we let $t(c)$ be the Dehn twist about $c$ (see p. 404 of [3]). Finally let $\left(n_{1}, \ldots, n_{g}, p\right)$ be $g+1$ distinct, pairwise relatively prime integers, each greater than two, with $p$ a prime. We set

$$
\Phi=\prod_{i=1}^{g} t\left(a_{i}\right) t\left(b_{i}\right)^{-n_{i}+1}
$$

and

Received by the editors June 23, 1977.

AMS (MOS) subject classifications (1970). Primary 30A46, 32G15.

Key words and phrases. Mapping class groups, Teichmüller modular group, Riemann surface, automorphism.

'The author gratefully acknowledges support from the Research Council of Rutgers University in the form of a summer fellowship.

(1) American Mathematical Society 1978 


$$
\Psi=\prod_{i=1}^{g} t\left(a_{i}\right)^{p} t\left(b_{i}\right)^{-p n_{i}+p} .
$$

Raymond and Tollefson [3] have shown that $N\left(\Phi_{\#}\right) /\left\langle\Phi_{\#}\right\rangle$ contains no elements of finite order. Here $\left\langle\Phi_{\#}\right\rangle$ is the subgroup of $M(g)$ generated by $\Phi_{\#}$. We will show how with minor modifications their argument also proves that $C\left(\Psi_{\#}\right)$ contains no elements of finite order (\$3). In $\$ 2$, we show that if $\Psi_{\#}$ is in the normalizer of an element of prime order, it is in its centralizer. The two conclusions taken together give the desired result.

2. Normalizers of elements of finite order. We use the following theorem:

THEOREM 1. Let $h$ be a homeomorphism of $F$ such that $h_{\#}$ is of prime order $q$. Let $t$ be the number of fixed points that $h$ would have on a surface on which it were conformal and $g_{0}$ the genus of the factor surface. There is an integral homology basis for $F$ consisting of homology classes of curves of the following form:

(i) When $t>0$ :

$$
\begin{array}{ll}
c_{i}, h\left(c_{i}\right), \ldots, h^{q-1}\left(c_{i}\right), & i=1, \ldots, 2 g_{0} \\
d_{j}, h\left(d_{j}\right), \ldots, h^{q-2}\left(d_{j}\right), & j=1, \ldots, t-2 .
\end{array}
$$

These curves satisfy the homology relations

$$
\sum_{k=0}^{q-1} h^{k}\left(d_{j}\right) \approx 0, \quad j=1, \ldots, t-2
$$

and no others.

(ii) When $t=0$ :

$$
c_{i}, h\left(c_{i}\right), \ldots, h^{q-1}\left(c_{i}\right), \quad i=1, \ldots, 2 g_{0}-2,
$$

$a$ and $b$.

These curves satisfy the homology relations $h(a) \approx a$ and $h(b) \approx b$ and no others.

Proof. See Theorem 1 and the proof of Theorem 2 in [2].

Let $H_{1}\left(F, Z_{p}\right)$ be the first homology group of $F$ with coefficients in the integers modulo $p$.

THEOREM 2. Let $f$ be a homeomorphism of $F$ such that $f_{*}$ induces the identity on $H_{1}\left(F, Z_{p}\right)$ for some prime $p$. Let $h$ be a homeomorphism of $F$ with $h_{\#}$ of prime order $q$. Then $f_{\#}$ is in $N\left(h_{\#}\right)$ if and only if $f_{\#}$ is in $C\left(h_{\#}\right)$.

Proof. We will prove $f_{*} \in N\left(h_{*}\right)$ implies $f_{*} \in C\left(h_{*}\right)$. Then from $f_{\#} h_{\#} f_{\#}^{-1}=h_{\#}^{r}$, we conclude that $f_{*} h_{*} f_{*}^{-1}=f_{*}^{r}$ and, thus, $h_{*}=h_{*}^{r}$. By Lemma 8 [3], this can only happen if $h_{\#}^{r}=h_{\#}$. Thus $r=1$.

Using the Riemann-Hurwitz relation $g=q g_{0}+(t-2)(q-1) / 2$, and the fact that $g \geqslant 3$, we obtain three possible cases. In each case we assume $f_{*} h_{*} f_{*}^{-1}=h_{*}^{r}$ and conclude $r=1$. We use the basis of Theorem 1 .

Case (i). $g_{0} \neq 0$ and $t>0$. Then $f h f^{-1}\left(c_{1}\right) \approx h^{r}\left(c_{1}\right)$. We calculate $f^{-1}\left(c_{1}\right)$ 
$\approx c_{1}+p z$ for some curve $z \cdot h\left(c_{1}+p z\right) \approx h\left(c_{1}\right)+p h(z)$ and $f h\left(c_{1}+p z\right) \approx$ $h\left(c_{1}\right)+p h(z)+p w$ for some curve $w$. Let $v=h(z)+w$. Then $h\left(c_{1}\right)+p v \approx$ $h^{r}\left(c_{1}\right)$. This says that we could replace $h^{r}\left(c_{1}\right)$ in the integral basis by $p v$. Since curves in a basis must be primitive, this cannot happen and we must have $v \approx 0$. Then $h\left(c_{1}\right) \approx h^{r}\left(c_{1}\right)$. But the images of $c_{1}$ under $h$ are distinct. Thus $r=1$.

Case (ii). $g_{0}=0$ and $t>2$. Arguing in a manner similar to case (i), we obtain $h^{r}\left(d_{1}\right)-h\left(d_{1}\right) \approx p v$ for some curve $v$. If $r \neq q-1$, then the same argument shows $r=1$. If $r=q-1$, we obtain $2 h\left(d_{1}\right)+p v+h^{2}\left(d_{1}\right)$ $+\cdots+h^{q-2}\left(d_{1}\right) \approx-d_{1}$. Note that we may assume $q \neq 2$. The homology relation says that we can replace $d_{1}$ in the basis by $p v$, which again gives a contradiction.

Case (iii). $t=0$ and $g_{0} \geqslant 2$. We use the same argument as in case (i).

Corollary 1. Assume $h_{\#}$ is of prime order $q$. Then $\Psi_{\#}$ is in $N\left(h_{\#}\right)$ if and only if $h_{\#}$ is in $C\left(\Psi_{\#}\right)$.

Proof. From Theorem 2, $\Psi_{\#}$ is in $N\left(h_{\#}\right)$ if and only if $\Psi_{\#}$ is in $C\left(h_{\#}\right)$. But $\Psi_{\#}$ is in $C\left(h_{\#}\right)$ if and only if $h_{\#}$ is in $C\left(\Psi_{\#}\right)$.

3. Modification of the Raymond-Tollefson argument. We let $P$ be the matrix of $\Psi_{\#}$ and $I$ the identity matrix of the appropriate dimension.

$$
P=\left(\begin{array}{ccccc}
X\left(n_{1}\right) & 0 & 0 & \cdots & 0 \\
0 & X\left(n_{2}\right) & 0 & \cdots & 0 \\
\vdots & & \ddots & & \vdots \\
0 & 0 & 0 & \cdots & X\left(n_{g}\right)
\end{array}\right)
$$

where $X(n)$ is the $2 \times 2$ submatrix

$$
\left(\begin{array}{cc}
1+p^{2}(n-1) & p \\
p(n-1) & 1
\end{array}\right)
$$

with $n>2, n$ and $p$ relatively prime.

LEMMA 1. The centralizer of $X(n)$ in $\mathrm{GL}(2, \mathbf{Z})$ is the subgroup of matrices of the form

$$
\left(\begin{array}{cc}
b(n-1) p+d & b \\
b(n-1) & d
\end{array}\right) \text { where } d^{2}+b d(n-1) p=b^{2}(n-1) \pm 1
$$

Proof. Calculation.

LEMMA 2. If $B \in \mathrm{GL}(2, \mathrm{Z})$ commutes with $X(n)$ and $B^{k}=I, k>1$, then $B= \pm I$.

Proof. Same as the proof of Lemma 5 [3]. 
LEMMA 3. If $m \neq n$, then $Y=\left(\begin{array}{ll}0 & 0 \\ 0 & 0\end{array}\right)$ is the only solution for $Y X(n)=X(m) Y$.

Proof. Calculation.

Let $A=\left(a_{i j}\right)$ be a $2 g \times 2 g$ matrix in $\operatorname{GL}(2 g, Z)$ in which the $a_{i j}$ are $2 \times 2$ submatrices.

LEMMA 4. If $A$ has finite order and $A P=P A$, then $a_{i j}=\left(\begin{array}{ll}0 & 9 \\ 0 & 0\end{array}\right)$ for $i \neq j$ and $a_{i i}= \pm I$ for $1 \leqslant i, j \leqslant g$.

Proof. Same as the proof of Lemma 7i [3].

COROLlaRY 2. The group $C\left(\Psi_{\#}\right)$ has no elements of odd prime order and any element of order two induces the identity on $H_{1}\left(F, Z_{2}\right)$.

Proof. If $f_{\#} \Psi_{\#} f_{\#}^{-1}=\Psi_{\#}$, then $f_{*} \Psi_{*} f_{*}^{-1}=\Psi_{*}$. By Lemma $4, f_{*}$ is an involution inducing the identity on $H_{1}\left(F, Z_{2}\right)$. But the map $f_{\#} \rightarrow f_{*}$ is one-to-one on elements of finite order (see Lemma 8 of [3]). Thus if $f_{*}$ is an involution, so is $f_{\#}$.

THEOREM 3. $\Psi$ is not homotopic to $f \Psi f^{-1}$ for any nontrivial involution $f$ of $F$.

Proof. This proof follows word for word the proof of Theorem C, p. 410 of [3]. Every property of $\Phi$ used either explicitly or implicitly in the proofs there of Theorem C and Lemmas 10-13 also holds for $\Psi$.

4. The example. Recall $\Psi=\prod_{i=1}^{g} t\left(a_{i}\right)^{p} t\left(b_{i}\right)^{-p n_{i}+p}$ where $\left(n_{1}, \ldots, n_{g}, p\right)$ are distinct integers, pairwise relatively prime, each greater than two with $p$ a prime.

THEOREM 4. If $g \geqslant 3$, then $\Psi_{\#}$ is not in the normalizer of any element of finite order in the mapping class group.

Proof. Since $\Psi_{*}$ induces the identity on $H_{1}\left(F, Z_{p}\right)$ and $\Psi_{*}$ is not the identity, $\Psi_{*}$ is of infinite order. Thus $\Psi_{\#}$ is of infinite order. Since a cyclic group contains a unique subgroup of every order dividing the order of the group, if $\Psi_{\#}$ normalizes an element of finite order, it must normalize an element of prime order. Let $f_{\#}$ be such an element. By Corollary $1, f_{\#}$ is in $C\left(\Psi_{\#}\right)$. By Corollary 2 and Theorem $3, C\left(\Psi_{\#}\right)$ contains no elements of prime order. Thus $f_{\#}=1$.

REMARK. Theorem 1 implies that if a nontrivial involution induces the identity on $H_{1}\left(F, Z_{2}\right)$, its matrix is $-I$. This fact could be used to simplify the proof of Theorems 3 and $\mathrm{C}$ of [3].

\section{REFERENCES}

1. J. Birman, Braids, links, and mapping class groups, Ann. of Math. Studies, no. 82, Princeton Univ. Press, Princeton, N. J., 1974.

2. J. Gilman, A matrix representation for automorphisms of compact Riemann surfaces, Linear Algebra and Appl. 17 (1977), 139-147.

3. F. Raymond and J. L. Tollefson, Closed 3-manifolds with no periodic maps, Trans. Amer. Math. Soc. 221 (1976), 403-418.

Departiment of Mathematics, Rutgers University, Newark, New Jersey 07102 